\title{
Community Perceptions of Indigenous Healers and Mental Disorders in Zimbabwe
}

\author{
Lazarus Kajawu $^{1 *}$, Manase Chiweshe ${ }^{2}$, Jacob Mapara ${ }^{3}$ \\ ${ }^{1}$ Department of Psychiatry, University of Zimbabwe, Harare, Zimbabwe \\ ${ }^{2}$ University of Zimbabwe, Harare, Zimbabwe \\ ${ }^{3}$ Chinhoyi University of Technology, Chinhoyi, Zimbabwe \\ Email: ^lkajaw@gmail.com, manasekudzai@gmail.com, jacobmapara@gmail.com
}

How to cite this paper: Kajawu, L., Chiweshe, M. and Mapara, J. (2019) Community Perceptions of Indigenous Healers and Mental Disorders in Zimbabwe. Open Journal of Psychiatry, 9, 193-214.

https://doi.org/10.4236/ojpsych.2019.93015

Received: April 11, 2019

Accepted: May 24, 2019

Published: May 27, 2019

Copyright (c) 2019 by author(s) and Scientific Research Publishing Inc. This work is licensed under the Creative Commons Attribution International License (CC BY 4.0).

http://creativecommons.org/licenses/by/4.0/

(c) (i) Open Access

\begin{abstract}
The World Health Organisation has made recommendations for partnerships between indigenous healing (IH) and biomedical therapy (BT) in the delivery of health services as a way of creating cultural sensitivity in mental health care (Bank, 2001). Yet, literature on prevalence, distribution, burden, and unmet needs for treatment of the mental disorders often exclude the role played by indigenous healing practitioners (IHPs). This study aimed to analyze mental health care from the perspective of communities on mental health care by IHPs to reveal their possible role in the surveillance studies of mental disorders in a settlement north-east of Harare in Zimbabwe through an exploratory qualitative methodology. Thirty in-depth interviews and three focus group discussions with key-informants were conducted to gather community perceptions of the nature of mental disorders treated by IHPs in Zimbabwe. Gathered data were coded using Constant Comparison Method with multiple members of the research team, enhancing validity and reliability. The results of the study reveal that while some patients presented with some mental disorders that were consistent with the BT diagnoses such as schizophrenia (Chirwere chepfungwa), depression (Kufungisisa), anxiety (Buka), post-traumatic stress disorder (Kurotomoka) somatisation (shungu), etc., other patients reported the disorders that were not recognised from a biomedical point of view such as the supernatural, cultural or social problems in IH. The findings were similar to the results of the first 17 world mental health surveys which show that the mental disorders are commonly occurring in all participating countries. This implied that the IHPs were treating common mental disorders reported in the low-income countries. More importantly, the IHPs treated a unique category which affected the majority of Zimbabwean patients. This study highlights the importance of the IHPs as complementary to BT in the management of the mental disorders. The IHPs should be included
\end{abstract}


in mental health surveillance with the BTPs in order to obtain realistic figures that enable proper planning and management of mental health disorders. Policies on integrating IHPs' work into mental health care must be drawn and published in national guidelines. More research is needed on integration guidelines.

\section{Keywords}

Indigenous Healing, Indigenous Healing Practitioner, Mental Health Care, Mental Disorders, Biomedical Therapy, Survey

\section{Introduction}

The state of the mental health disorders in global settings is alarming [1]. The high prevalence has created impetus for WHO to encourage the member countries to exploit all the resources, including the indigenous healers (IHPs), to fight the disease burden [2]. However, statistics on mental disorders are only derived through surveillance in bio-medical Therapists (BTPs). Unfortunately, no statistics on mental disorders (MDs) have been included from IHP because their work has is under-studied. Consequently, data on the surveillance of the common mental disorders, are poorly represented. To achieve effective surveillance for proper management of common mental disorders, the work by IHPs on mental disorders in resource poor countries has to be understood [3]. This paper, therefore aimed at analysing the community perceptions of healers and the nature of mental disorders they treat in Zimbabwe.

Although IHPs are ubiquitous in Zimbabwe, very few academics or BTPs know and understand their role in mental health care because of the secrecy due to lack of rigorous studies on their sociocultural practices in Zimbabwe [4]. The literature on disease burden in the global world; the African settings, including Zimbabwe, can help to illuminate on mental disorders IHPs treat. Currently there are no statistics from IHPs. This paper will explore literature onthe nature of mental disorders in the resource poor countries in order to explore the mental disorders found in settings where indigenous healers operate in, who ironically, serve $80 \%$ of the populations for their mental health treatment in the same regions [5]. First, the paper presents definitions of key concepts in the study, beginning with indigenous healing practitioner.

There is no one definition of an indigenous healing practitioner in Africa [6]. How an indigenous healer is defined depends on the social, cultural and spiritual background of the person [7]. An indigenous healer may be seen as a person who is recognized by the community in which he/she lives as competent to provide the health care by using the vegetable, animal, and mineral substances and certain other methods based on the social, cultural and religious backgrounds, as well as the knowledge, attitudes and beliefs that are prevalent in the community regarding the physical, mental and social wellbeing and the causation of the dis- 
ease and disability [8]. IHPs include in their treatment, mental disorders.

The mental disorders include a wide range of the mental health problems that present in the primary care as an important source of disease burden worldwide, including in Zimbabwe [9]. The most burdensome problems are the common mental disorders, including the anxiety, depression, post-traumatic stress disorder and substance abuse, and to a lesser extent "the severe long-term health disorders" such as schizophrenia and dementia [10]. Although the primary care has an important role to play in management of more severe disorders, the common mental disorders are generally viewed as the main remit of the primary care [11].

Currently, there is an explosion of mental disorders the world over, especially in resource poor countries, including Zimbabwean [12]. While there is proper surveillance in advanced countries to monitor the occurrences of mental disorders, in Zimbabwe and other resource poor countries, surveillance is based on statistics obtained through BTPs and the information excludes the work by IHPs, who are accused of not being scientific [13]. Unfortunately, people are reluctant to use the formal health services for their mental health care, accusing the formal health care of being culturally insensitive. Instead, many people with mental disorders consult the IHPs who are unfortunately little studied and so much data on mental disorders is therefore lost [14]. To establish accurate data aimed at providing information to mental health policy makers about the treatment of the common mental disorders, the work by IHPs on mental disorders in resource poor countries has to be understood [15]. This is because $80 \%$ of the populations in these regions use IH for their mental health treatment, and any meaningful work intended to gauge the size of the mental health problems has to include the IHPs who attract more patients [16]. First, a pilot study on the nature of the mental health problems seen by the IHPs has to be established. In order to address the global burden of mental health issues in low-income countries (LICs), the World Health Organization has called for the optimization of all available resources to bolster the delivery of mental health treatment in primary care [17]. In Zimbabwe, many studies have looked at IH in general [7] [12] [13] but not much has been done on mental health treatment [18]. Once the information is known, it can be used to inform future studies that help to inform policy makers on the management of mental disorders.

Although little work on $\mathrm{IH}$ therapies have been formally evaluated as mental health treatments [18], their work in mental health care is not known [19]. Furthermore, it is not known how many patients consult $\mathrm{IH}$, or the nature of their mental health problems. Therefore, evidence from this research will be useful toinform future studies in order to help policy makers about the prevalence, distribution, burden, and the unmet needs for the treatment of the common mental disorders and plan the provision of mental health service in Zimbabwe to reduce the burden of disease. The author therefore anticipates that the evidence that will be gathered by this study will contribute invariably to decisions regarding the exploitation of $\mathrm{IH}$ in mental health work to improve on the accuracy of surveil- 
lance on mental disorders in Zimbabwe. Furthermore, the research will act as a reference for future programmes guided by evidence on the ground.

\section{Literature Review on State of Mental Health: A Reflection of IHPs' Work}

\subsection{Introduction}

The literature is on the prevalence, distribution, burden, and the unmet needs for the treatment of the common mental disorders and the state of interventions, skills and capacity for mental health care in the resource poor countries. The literature will reflect on the nature of mental disorders seen in resource poor countries which IHPs also see but are not currently reported on because IH is informal [3]. We begin by making an overview of the global mental disorders.

\subsection{Overview of Global Mental Disorders in the Resource Poor Countries}

The United Nations General Assembly recognized for the first time the mental health and substance abuse as the global priorities inrecent findings from the WHO World Mental Health (WMH) surveys on the global burden of mental disorders [1]. The WMH surveys are representative community surveys in 28 countries throughout the world aimed at providing information to mental health policy makers about the prevalence, distribution, burden, and the unmet needs for the treatment of the common mental disorders [3].

The study found that the mental disorders are commonly occurring in the general population, often have an early age-of-onset, and often are associated with the significant adverse societal costs [20]. The first $17 \mathrm{WMH}$ surveys ${ }^{1}$ show that the mental disorders are commonly occurring in all the participating countries (WHO, 2013). Many mental disorders begin in childhood-adolescence and have significant adverse effects on subsequent role transitions in the WMH data [3]. Adult mental disorders are found to be associated with such high role impairment in the WMH data that available clinical interventions could have positive cost-effectiveness ratios (Steel et al., 2014b). Similar findings obtain in African countries including the state of interventions, facilities available, skills and capacity for mental health care.

\subsection{Mental Disorders in the African Context}

Mental health in Africa will be reviewed under the widely held view that; mentally ill patients brought the disease upon themselves; and by using illicit drugs may be one reason African governments do not prioritize mental health [19]. Experts have also pointed to a tendency in Africa to view acute mental health diseases as supernatural afflictions that can be cured only through spiritual or ${ }^{1}$ Some of the participating countries are Colombia, Mexico, United States, Nigeria, South Africa, Lebanon, Belgium, France, German, Israel, Italy, Netherlands, Spain, Ukraine, Japan, and New Zealand. Zimbabwe is non-participant country. 
traditional medicinal interventions [8] [9] [21]. This situation has created a big gap in the delivery of the mental health care and there is need for research in collaborative healing between IH and BT in mental health.

Fortunately, in September 2015 the United Nations General Assembly as reported above, included the mental health and the substance abuse in the global Sustainable Development Goals, marking the first-time world leaders recognized mental health as a global priority [22]. African countries can begin to act on this recognition by increasing their spending on the mental health by including use of the IH; currently the African countries dedicate on average less than $1 \%$ of their health budgets (themselves minuscule) to mental health, compared with $6 \%-12 \%$ in Europe and North America [19]. With this in mind, the paper presents some reflections of mental health pictures in some African countries.

The health experts estimate that a fourth of the Kenyan population of 44 million suffers from a range of the mental diseases, including the schizophrenia and the other psychotic disorders, bipolar disorder, depression and the severe anxiety [23]. However, Kenya has only about 80 psychiatrists and 30 clinical psychologists, fewer than its 500 psychiatric nurses, of which only 250 work in the mental health (Husain et al., 2016a). According to the World Health Organization (WHO), yet the country spends only about $0.05 \%$ of its health budget on mental health [24]. About $70 \%$ of the mental health facilities in the country are located in the capital, Nairobi [25]. This leaves the majority of the population without care, and naturally seeking care outside the formal health system in the IH services.

The picture is slightly different in South Africa. Out of a population of 53 million, one-third are afflicted with the mental diseases, according to experts [26]. However, while the country boasts of 22 psychiatric hospitals and 36 psychiatric wards in the general hospitals, only about $14 \%$ of the population have access to the facilities due to the problems of inequality and the majority of about $75 \%$ of mentally ill South Africans without access to psychiatric or therapeutic care, or turn to the informal health sector for their treatment [27]. Similarly, the disorders like depression, anxiety and schizophrenia are common in Nigeria, as in other countries in Africa [28]. While both South Africa and Kenya have more psychiatrists per capita, as well as more psychiatric beds per capita, the oil-rich Nigeria offers a more dismal picture. The WHO estimates that fewer than $10 \%$ of mentally ill Nigerians have access to a psychiatrist or health worker, because there are only 130 psychiatrists in the country of 174 million people and that the number of mentally ill Nigerians ranges from 40 million to 60 million [29].

Ghana shares a lot with other African states in relation to mental health care. The country has three psychiatric hospitals and about 20 psychiatrists [26]. A report by Human Rights Watch (HRW), a non-governmental organization, estimates that 2.8 million Ghanaians (out of a population of 25.9 million) has mental illness [26]. The HRW report mentioned that drug-related psychosis affected $8 \%-10 \%$ of all mental patients, while $20 \%-30 \%$ of patients were diagnosed with schizophrenia, $20 \%$ with bipolar disorder, and $15 \%-20 \%$ with major 
depression. Sadly, 97 out of 100 mental patients who need health care have no access to these services [30]. However, Ghana has distinguished itself so far. In 2012, Ghana took a significant step forward in addressing the nation's mental health when it passed Act 846, also known as the Mental Health Act, becoming one of the few countries in Africa to set out a mental illness policy [31]. While the mental health picture is bad in politically stable economies such as Kenya, Nigeria, Ghana, and others, the mental health picture is far worse off in poorer countries especially those that have recently experienced wars, or conflicts, such as Liberia and Sierra Leone [20]. Zimbabwe is no exception from this group and requires attention.

\subsection{Mental Disorders and the Available Facilities, Skills and Capacity for Mental Health Care in Zimbabwe}

In Zimbabwe mental disorders are commonly occurring in the general population, are on the rise, often have an early age-of-onset, and often are associated with significant adverse societal costs [32]. Zimbabwe went through two decades of economic melt-down and this has had a devastating mental, emotional and physical toll on the generality of Zimbabweans who can no longer absorb the rigours of the meltdown [33]. World Health Organisation (WHO), reports that some 1, 3 million of the country's 14 million people, representing 10 percent of the population, have one form of a mental disorder or another [34]. This is quite a huge increase, the figure has more than doubled in less than 20 years, considering that there were just above 600,000 mentally ill patients in 1999 [35]. Unfortunately, the increase in the numbers of mentally ill people does not match the staff complement in the country. WHO estimates show that Zimbabwe has only 14 psychiatrists to cater for the 1,3 million mental patients, translating to a doctor to patient ratio of 0,08 percent per 100,000 people? In addition, there is a shortage of mental health facilities, including staff and drugs, across the country. The whole country has only 20 registered clinical psychologists and nine public mental health institutions [33].

A couple of factors point to a gloomy picture of Zimbabwean situation: the country's economy keeps on shrinking; Zimbabwe is currently grappling with a record unemployment, now believed to be over 90 percent, owing to the closure of companies and widespread retrenchments; Even for those who are gainfully employed, very few are earning salaries that are consistent with the breadline, estimated at about US $\$ 550$ per month; the majority of employees outside the public sector are toiling for months on-end without pay; at least 120,000 workers across the country worked without pay between 2015 and 2016; An estimated 350,000 formally employed workers have sunk into abject poverty; and more than 80 percent of Zimbabwe's population has been driven into the deepest depths of poverty [34]. In addition, The 2015 Zimbabwe Poverty Atlas estimates that 96 percent of the country's population in the countryside is now living on less than a dollar a day, while poverty in the major two cities of Bulawayo and Harare stands at 37.2 percent and 36.4 percent respectively (Chibanda et al., 
2016). Overwhelmed by the situation, some families are neglecting mentally ill relatives by condemning them into psychiatric hospitals [36].

Studies suggest that there are high rates of psychopathology in Zimbabwe [37]. For example, even in the 1990s and prior to the worst of the decline in Zimbabwe, local studies have shown that the prevalence of psychological disorders such as depression and anxiety varied from $10.5 \%$ in primary care [38] to about $26 \%$ in rural and urban settings and $41 \%$ at two primary clinics in the suburbs of Harare [18]. In another study, 205 late term pregnant women were screened for mental disorders. Of these, 44 (46\%) of 95 women who were considered as high risk and $9 \%$ of 110 women who were considered low-risk met criteria for depression [39]. The Prevalence of depression in the rural community near Harare, the capital city of Zimbabwe, was 31\% [40]. About 10\% - 20\% of patients from other provinces and health centres suffered from common mental disorders which included anxiety and depression [39]. The main challenges that mental patients face include among other things, shortage of mental health specialists, limited care and support and culturally insensitive mental healthcare. WHO representative in Zimbabwe, David Okello, has described the situation as a "crisis", which calls for urgent measures to reverse it? However, the abundantly available local resources of indigenous healers carry the bulk of the burden in terms of managing the mental disorders behind the scenes because their contribution is not recognised in the scientific community, and valuable information on the magnitude of mental disorders is lost. This is the gap that this paper tries to fill.

\subsection{Conceptual Framework}

This paper used the following four theoretical frameworks, to guide the study involving indigenous healing approach: health belief model (HBT), reasoned action and planned behaviour model (RA + PBT), indigenous knowledge systems (IKS) and explanatory model of illness (EMI). These theories helped to justify why some people opt for indigenous practices for their mental health care. These theories explain why the majority of people in Zimbabwe opt for indigenous healing practices. In trying to justify on the use of these theories in the present study, the paper briefly looked at the main features of each theory followed by a review of strengths and weaknesses of the approach and the paper exploited the strengths of each theory. The HBT is a psychological health behaviour change model developed to explain and predict health-related behaviours, particularly in regard to the uptake of health services [41]. The Health Belief Model (HBT) was developed in the early 1950 s by social scientists at the U.S. Public Health Service in order to understand the failure of people to adopt disease prevention strategies or screening tests for the early detection of disease. Later uses of HBT were for patients' responses to symptoms and compliance with medical treatments. The models were all useful in explaining the choice made by people to visit or not, IHPs for the treatment of mental disorders. 


\section{Methods}

This study used a sample of 30 patients of IHPs who were complaining of mental health problems to the IHPs for one on one interviews and 18 members from the community for a focus group discussion, each with six members making a total of 48 participants, from June, 2018 to $31^{\text {st }}$ December, 2018. These were calculated using the information in Glaser \& Strauss, 2009 and were enrolled in the study, representing people with mixed views and came from the different regions of the country to settle in the seven wards of the Epworth community [42]. This was done to minimize the sources of error in the subject selection and the comparability [43]. A qualitative design was selected because this was appropriate in this case because little was known about the IH and a qualitative approach would allow for a deep, textured exploration of the perspectives on mental disorders in Zimbabwe [44].

In this study, the understanding of the nature of mental health work by IHPs emerged from the interviews with the participants and the direct observations of the IH practices. The study chose a case study because IH was of special interest and dealt with unique issues in the management of mental health [45]. This would help to understand the role of IH in the treatment of mental disorders within the local settings. The study was conducted in Epworth, a large peri-urban habitation located 16 kilometres north-east of Harare. Epworth community is of very diverse cultural composition. A non-probability sampling method which is purposeful sampling was used to recruit members of the community while the snowballing was used to recruit the patients of the IHPs [43]. The participants were selected for the study if they spoke any of the indigenous language in Zimbabwe (i.e. Shona or Ndebele) and if they were 18 years of age or above. The participants were excluded from the study if they were minors (under 18 years of age) including those with severe cognitive impairments.

The researcher requested the IHPs for their permission to let the researcher recruit the patient participants at their healing shrines. To recruit the eligible patients, the researcher used the snowballing sampling strategy by first approaching one patient who had seen an IHP and requested them to link him to the next patient they knew had consulted an IHP. The investigator explained the study objectives, procedures and obtained the informed consent from all the participants. The community members were recruited at the social gathering sites; such as food distribution depots, using a convenience sampling strategy as they were leaving the site. The investigator explained the study objectives, the procedures and obtained the informed consent from all the participants.

From January of 2018 to December of 2018, the researcher conducted the interviews in Epworth. The researcher made use of a number of data collection methods which included 30 in-depth interviews (IDI), three focus group discussions (FGDs) and observation for triangulation purposes. Two research assistants helped with notes taking and audio recording. The researcher started collecting data by conducting the in-depth interviews with the patients of the IHPs in the mental health work focusing on the experiences of patients when they vis- 
ited the IHPs, their mental health problems treated by IHPs and why they preferred the treatment of IHPs to BTPs work followed by FGDs. During the study period, the researcher made observations on the indigenous healing approach when interacting with the participants.

IDI were used because the researcher wanted detailed information about a person's thoughts and behaviours or to explore the new issues in depth [46]. The interviews are often used to provide the context to the other data (such as the outcome data), offering a more complete picture of what happened in the program and why [47]. In this case, this study, explored the experiences of patients when they visited the IHPs and why they preferred the IHPs to BT specialists using the IDI. The IDI was used with patients of the IHPs in place of the focus groups because the patients consulting the IHPs might have not been comfortable talking openly in a group. In addition, the researcher preferred to distinguish an individual (as opposed to a group) opinions about the IH approach. The IDI was conducted with the 30 patients of the indigenous healers, using the semi-structured interview guides and the patients were chosen because they would give their perceptions on the use of the IH in the treatment of the mental disorders in Zimbabwe. IDIs were conducted in the IHP's healing shrine and each interview lasted about an hour.

For the focus groups interviews, the interviewer used the guide to keep the interactions focused while allowing individual perspectives and experiences on $\mathrm{IH}$ to emerge [48]. Groups were typically made up of 6 people with similar backgrounds who participated in the interview for one hour. In this study, three series of different focus groups were conducted to get a variety of perspectives and increase confidence in whatever patterns of indigenous characteristics emerged [47]. The researcher engaged two research assistants to help conduct the groups so that one person focused on facilitating the group while the other took detailed notes and dealt with mechanics such as the tape recorders, cameras, and any special needs that arose, for example, someone needing to leave early or becoming overwrought [43]. Even when the interview was recorded, good notes helped in sorting out who said what when the tape recording was transcribed. The focus group interviews had several advantages as noted by Krueger, (1994:48).

Additionally, in order to tap into issues that otherwise were hidden from insights of others, the paper, in addition to interviews and focus group discussion, the researcher was involved in non-participant observations during the whole therapy process which lasted for about one hour and made thick descriptions of the whole process of the IH using an observation check list with information discussed above [49]. The researcher also observed himself, in terms of his reactions during all the encounters [50]. This method was used to gain the first-hand information on IHPs' practices and the nature of mental disorders seen by IHPs and to triangulate the information with the other data obtained from the patients of IHPs; including the members of the community [43]. The in-depth and focus group discussion interviews were conducted in Shona, audio recorded, professionally transcribed, translated into English, and back-translated to Shona 
to check for consistency. The researcher pilot-tested the instruments using the interview guides and observational checklist. Data was collected from 5 IHPs, 5 patients of IHPs and two focus group discussion sessions; one with 6 members of the community and another session with the 6 nurses for the pilot study. After obtaining data from the respondents, the researcher then made some improvements to the interview guides and observational checklist (Huberman \& Miles, 2002). The pilot study enabled the researcher to test and fine tune the interview guides, procedures, and observational skills to determine the final sample.

Data were analysed using constant comparison method. The actual process of the data analysis usually took the form of clustering the similar data. In this study data was transcribed from the audio recorders and themes were developed from the transcripts. The analysis of the data was guided by the objectives.

The study was approved by the Chinhoyi University of Technology and the Medical Research Council of Zimbabwe (MRCZ). Approval was obtained from Kunaka District Hospital in Epworth and the ZINATHA before the start of the study. Informed consent was obtained from all the participants, including permission to audio-record the interviews.

\section{Findings and Discussion}

\subsection{Introduction}

The aim of the paper was to explore the community perceptions of IHPs and the mental disorders treated by IHPs in Zimbabwein order to inform policy makers about the prevalence, distribution, burden, and the unmet needs for the treatment of the common mental disorders in Zimbabwe and other resource poor countries of the world. The results from the thick descriptions made from the direct observations by the investigator were synthesized with the responses from the in-depth interviews of the indigenous healers' patients and members of the community. First, the paper presents the demographics for the study groups.

\subsection{The Participants' Demographics}

\subsubsection{The Sample Characteristics of All the Key Informant Interview Participants}

The paper recruited a total of 48 key informant participants, 30 patients of the IHPs, $70 \%$ women and $30 \%$ men at the IHPs' shrines, and 18 participants for the three focus group discussions, each with six people (Table 1). The mean age for the participants was 43 years and the mean number of years of formal education was nine. All of them were Shona-speaking. Of the 51, participants approached, 48 accepted to take part as the key informants; 3 declined because they were too busy at the time and one was cognitively impaired.

\subsubsection{Sub-Samples' Characteristics of the IHPs, the Patients of IHPs, Community Members and Nurses}

For the sample of the patients of the IHPs, there was a total of 30 patients, 20 women and 10 men at the IHPs' shrines. The mean age for the 30 patients was 
Table 1. Sample characteristics.

\begin{tabular}{ccccccc}
\hline & No & $\%$ Males & $\begin{array}{c}\% \\
\text { Females }\end{array}$ & Range & $\begin{array}{c}\text { Mean } \\
\text { education }\end{array}$ & $\begin{array}{c}\text { Language } \\
\text { Spoken }\end{array}$ \\
\hline $\begin{array}{c}\text { Patients' } \\
\text { sub-sample }\end{array}$ & 30 & 30 & 70 & 18 yrs - 56 yrs & 10 & Shona \\
$\begin{array}{c}\text { Community } \\
\text { members' sub-sample }\end{array}$ & 18 & 33 & 67 & 70 yrs - 21 yrs & 11 & Shona \\
\hline
\end{tabular}

45 years and the mean number of years of education was 10. Eighteen (21 women and 9 men) people were drawn from the community members, for the three focus group discussions, each with six people at the social amenities' points. The mean age for the 3 groups was 36 years and the mean number of years of education was 11 . The mean age for the group was 40 years and the mean number of years of education was 15 years. The study participants were similar to the people who normally consult in $\mathrm{IH}$.

\subsection{Community Perceptions of Ihps and Nature of Mental Disorders in Zimbabwe}

\subsubsection{Introduction}

From the observations made during the study period, the researcher noted that the patients consulted many different types of indigenous healers for their treatment of mental disorders similar to the results of the study by Patel and others [39] in Zimbabwe or what Robertson in [51] found in South Africa, or in Nigeria [52], or for Sudan in [53]. These were mainly the herbalists, the spirit mediums, the diviners and the faith healers. Although faith healers were somewhat different from the other service providers, they were classified as the IHPs in this paper because of their similarities in their operations with the rest of the IHPs [8]. Data suggests that there are different IHPs in the treatment of mental disorders in Zimbabwe. In addition, each of the different healing orders carried a specialized role in the treatment of the mental disorders. The researcher noted that many herbalists (5 out of 6), often called vana godobori (indigenous healers), reported that they were spiritually called to their profession, as they just received and administered the healing information in different ways on their patients However, therapy was terminated when the client feels well [9]. Many herbalists (godobori) (4 out of 6) reported they shared some of the functions of the diviners, but their concern was mainly with the healing of the illnesses. They prepared the concoctions, some of which had perceived protective value to ward off the evil spirits similar to the results of an international study in the United States of America [54]. For example, a 26-year-old male indigenous healer with 6 years' experience had this to say;

When a patient with (buka) anxiety disorder-like symptoms comes for the treatment, you mix the herbs and ask the patient to rub a little bit of the herbs on the fore-head to repel the evil spirits (Herbalist).

This implied the IHPs lacked an organised system of the measurement of their 
herbs and the practice was likely to be a source of conflict which would create some problems in the management of the mental disorders, if the issue was not resolved.

Unlike the herbalists, many spirit mediums (masvikiro) (4 out of 6) mentioned they were consulted for their guidance, the management of the misfortune and the treatment of mental illnesses (Berg, 2003). They reported that they were "called" to their profession and were foretellers of the droughts, the diseases and the major events. They received the revelations from their ancestral spirits [55]. One 85-year-old male spirit medium (svikiro) seeing a patient with addiction problems observed; "The spirit shows me the patient is losing his mind through the abuse of alcohol (kurasika pfungwa nezvinodhakwa)" (Spirit medium). The spirit mediums were useful in providing motivation therapy for those with alcohol abuse. The researcher also observed that a diviner carried a similar function to that of the spirit medium. The divination in Un'anga (IH) was a combination of astragyromancy, which was a method of reading dice marked with various symbols, numbers and letters, and cleromancy, or divination by lots, which was also called the interpretive divination [56].

In contrast to the above different groupings in the $\mathrm{IH}$, the researcher noted that all the faith healers (6 out 6 ) each belonged to a religious healing group. Unlike the indigenous healers who fell in the distinct healing orders, the researcher noted that all the faith healers from the Apostolic, the Pentecostal or the Independent churches referred to themselves as muporofita (faith healer), which like n'anga (IHP) addressed both the physical and spiritual ailments as well as exorcising the evil spirits [57]. The patients reported that the faith healers were helping them effectively. While a traditional healer (n'anga) sought for a solution, which acceded to the demands of the spirits, a faith healer's solution was based on a belief in the healing power of the Christian God, which was above all other powers. The researcher observed that in addition to their different methods of diagnosis, a faith healer (muporofita) downplayed the role of the ancestral spirit (mudzimu) and rejected the indigenous and the biomedical medicine. Despite these differences, the faith healers (maporofita) were still grouped as the indigenous healers ( $n^{\prime}$ anga) and were required to register with the Zimbabwe National Traditional Healers' Association (ZINATHA) [58]. The researcher found that the boundaries between the indigenous healers ( $n$ 'anga) and the faith healers (muporofita) were extremely fluid. Many faith healers (maporofita) incorporated the ancestral spirits (midzimu) and prescribed water or stones' therapy and numerous parishioners sought treatment from the clinics [8].

In religious healing, the faith healers mentioned they were responsible for the healing of the patients. The faith healers mentioned the religious healers in Zimbabwe were mainly influenced by the Christian missionaries, Arabic or Islamic teachings and indigenous cultures [59]. For example, the Zionist or African Apostolic Faith churches followed the customs of the indigenous rural society, but they had abandoned certain role boundaries and authority structures. 
All the faith healers mentioned that their difference with the indigenous healers was that their healing spirit was that of Jesus Christ and the Lord. For example, a 36-year-old female faith healer noted:

Only the Lord Jesus may direct us to pray for the patient's mental problem to go, or some water is given to the patient and the patient is freed from the evil spirits and blessed by the Lord (Faith Healer).

However, many faith healers mentioned that a few healers might practice witchcraft, in which case they were classed as witches. These were harmful people driven by the bitterness to hurt others [55]. They could be witches if they used their abilities for a wrong reason, such as killing people. The IHP's job in the past was to identify "who was the witch."

This was before the Witchcraft Suppression Act that prohibited folks from calling out a witch directly [9]. Their role was to identify this person but over time, the colonial government mislabelled them as witch doctors and witches themselves. This reflected the polarisation of the two approaches with a bearing on the current negative tendency to refuse the adoption of IHPs as mental health providers (Sodi et al., 2011). In spite of the wrong labels of a witch doctor or a witch, many patients (25 out of 30) reported that they could choose to consult the indigenous healers for the different reasons [55]. This indicated that the patients had a strong belief and conviction in the IH system which in turn had a bearing on what should be considered in modifying mental health therapy for the majority of the patients in Zimbabwe [13]. There were, however, some similarities among the functions of the IHPs. All the IHPs were focused on the healing of a patient by dealing with a problem spirit on the patient. The diviners, spirit mediums and the faith healers all focused on identifying the causes of mental illness, and the majority of the IHPs had an assistant. These IHPs treated patients suffering from different mental conditions discussed below.

\subsubsection{The Mental Health Disorders Seen by the IHPs in Zimbabwe}

The IHPs and their patients reported that while some patients (17 out 30) presented with some mental disorders that were consistent with the BT diagnoses, the other patients (14 out of 30 ) reported the disorders that were non-existent in the BT. Mental disorders that were consistent with the BT diagnoses were the schizophrenia (Chirwere chepfungwa), depression (Kufunganya), anxiety (Buka), post-traumatic stress disorder (PTSD) (Kurotomoka) somatisation (shungu), bipolar disorder (mhengera mumba), epilepsy (tsviyo) (Epilepsy is a physical disease in BT but the majority of the Zimbabweans perceived it as a mental disorder), personality disorder (kuzhangandira) and the substance abuse disorder (kuraradza rezvinodhaka), among others. The findings were similar to the results of the first $17 \mathrm{WMH}$ surveys which show that the mental disorders are commonly occurring in all the participating countries (WHO, 2013). This implied that the IHPs were treating the common mental disorders similar to those reported in the low income countries [1].

Data suggested that the IHPs were unique since they treated some mental dis- 
orders that were non-existent from the point of BT diagnosis. In addition, IHPs were complementing BTPs in treating common mental disorders. However, there was a danger for IHPs to treat epilepsy as a "spiritual issue" since it is a medical condition which requires specialists' services. There is a need to provide the IHPs with right information so that they refer people with this condition to the specialists. Nevertheless, the findings appear to corroborate many other studies done in low income countries which demonstrate the role of IHPs in the treatment of the common mental disorders [8] [9] [39].

However, from a biomedical point of view there was not a category to classify the other kinds of the diagnosis made by the IHPs similar to the results of an Australian study [60].

\subsubsection{The Mental Disorders Seen by IHPs: Nocategory to Classify the Same Diagnosis from a Biomedical Point of View}

Almost all the IHPs (23 out 24) reported seeing patients who were presenting with the mental disorders where there was not a category to classify the kinds of the diagnosis made from a biomedical point of view [56]. These included the supernatural, cultural or social problems in IH.A few IHPs (4 out 24) reported that they had seen some patients who had some mental conditions that had not healed after visiting the biomedical care providers. This was noted by a 67 -yearold female diviner:

This patient had mamhepo (bad airs) and had seen several doctors before but was not cured because her mental condition was not understood in biomedicine (Zvechivanhu zvisinganzwisisike) Diviner.

This category that was unique to the IHPs which was a dominant issue among the majority of the Zimbabwean patients but the BT specialists were not able to handle [8]. This emphasised the role of IHPs in any program that considered effective management of mental disorders in Zimbabwe.

1) witchcraft (zvehuroyi) in IH

All the IHPs (24 out of 24) perceived witchcraft as arising from evil minded people who were jealous and harmed others with magic [59]. Many faith healers (4 out of 6) mentioned a person suffered from a mental disorder when he or she was bewitched, for example, some patients developed some confusion (kutenderera musoro) after they were beaten by some witchcraft birds (zvishiri) or goblins (zvidhoma), which were mystical creatures used in witchcraft to harm people viewed as enemies [9]. This was noted by a 44 -year-old female faith healer; "I can tell how a patient fell ill through witchcraft (kuroyiwa) and developed confusion (vakadhanganyika musoro) and madness (kupenga)" (Faith Healer). This concept of witchcraft caused some problems in counselling, for example, in using cognitive behaviour therapy (CBT), witchcraft was considered a dysfunctional belief [61]. In contrast, witchcraft was real among the majority of Zimbabweans and could notsimply be dismissed as a dysfunctional belief. This made CBT irrelevant among the majority of the Zimbabweans who believed in witchcraft and the patients consulted in IH instead of BT. The beliefs in 
witchcraft calls for modifying therapy in order to include the cultural nuances which makes it culturally appropriate. The same applied to aggrieved spirits.

\section{2) Aggrieved spirits (Ngozi) in IH}

Many IHPs (22 out of 24) reported seeing the people they perceived were being tormented by ngozi (aggrieved spirits) and manifested the psychotic symptoms such as hallucinations, delusions etc. [62]. This was observed by a 39 yearold male spirit medium:

I treat a person who falls (anodonha), has bad airs (mamhepo) and exorcise aggrieved spirits (kutanda ngozi) and those patients molested by the evil spirits (vanoshungurudzwa nemweya yetsvina) (Spirit medium).

The concept of supernatural powers, for example, Ngozi, was still deep rooted among the majority of Zimbabweans and skills such as exorcism and other rituals were central to their resolution.

\section{3) Culturally defined trauma symptoms (e.g. Rukaho) in IH}

Most patients (26 out of 30 ) often understood their illnesses in a culturally specific way that did not correspond with the biomedical explanations of illness [9]. They discussed illnesses that were not considered pathological in biomedicine, and only trusted IHPs with their care. For example, many patients (20 out of 30) mentioned they had zvechivanhu (some culturally defined trauma symptoms), such as rukaho, a condition which was not understood in BM. A 45-year-old female patient observed;

If a person is having an affair with someone's wife, the wife's husband may use rukaho (black magic to fix the perpetrator). The victim fails to remove his penis from the vagina after sexual intercourse, and the partners committing the sexual offense can only be separated by IHP (Patient).

The existence of such conditions among the majority of Zimbabweans highlights the importance of IHPs as complementary to BTPs in management of the mental disorders among the majority of the Zimbabweans.

\section{4) Some interpersonal problems (e.g. Munyama) in IH}

Almost all the IHPs (28 out 30 ) reported treating some mental conditions that arose from some interpersonal problems. For example, many IHPs (25 out of 30) reported treating munyama (equivalent to bad luck, which was perceived to either prevent a person from getting a new partner or marriage, or when they had been rejected by either their boyfriend or husband) which resulted in mental problems. One 48-year-old female diviner noted;

"I treat those with relational conflicts (vanonetsana nezverudo); family related issues (matambudziko emhuri) and abuse (kushungurudzana)" (Diviner). The IHPs had a special role of dealing with the interpersonal problems which resulted in the mental disorders.

Data suggests that IHPs were playing a unique but indispensable role which was complementary to BTPs in treatment of mental disorders where they treated mental health issues that were not a category to classify the kinds of the diagnosisfrom a biomedical point of view. Therefore, the IHPs should not be side-lined as therapy was not complete without the input from IHPs. A diligent search in 
the literature revealed no similar result. In addition, there was no suggestion that any one type of a healing order specialized on one type of a mental disorder as the patients simply went to an IHP depending on the focus of treatment i.e. whether they wanted to know the causes of their problems, in which case they went to a spirit medium or diviner or a faith healer; or simply wanted herbs for treatment, and went to see an herbalist [18].

The knowledge gained from the findings contribute immensely to the management of the mental health burden in Zimbabwe and other resource poor settings, at least in terms of the high numbers of patients seen, and participants in this study expressing satisfaction with the care they received.

\section{Conclusion}

The paper has revealed that IHPs are dealing with special issues which BTPs are not addressing, together with common mental disorders with little or no recognition from the scientific community and BTPs. Unfortunately, large amounts of data are not included in national surveillance, leading to improper decisions due to skewed data in favour of BT source regarding the management of mental disorders, because work from IHPs is not recognised. However, the patients present with both conditions, recognised in BT and not recognised. The paper argues that IH approach should be adopted in the management of mental disorders in a complementary way, with IHPs specialising on the issues which normally are not addressed by BTPs, for example, cultural/spiritual problems., at least in surveillance studies to guide policy in the fight against disease burden and to inform policy makers about the prevalence, distribution, burden, and unmet needs for the treatment of the common mental disorders in Zimbabwe and other resource poor countries. More research is required to facilitate the adoption of IHPs into mental health work towards holistic therapy.

\section{Recommendations}

\subsection{Practice}

Training workshops and seminars on mental disorders should be organised in Zimbabwe for IHPs and BTPs to learn from each other on the nature of mental disorders. The workshops will help the mental health providers from the two approaches to appreciate each other's input in mental health care. It is envisaged that the health providers will then understand what each approach does and therefore appreciate the role of each approach in mental health care which will assist in accuracy of measurement of mental disorders. The nurses and other mental health providers should embrace IHPs in order for them to incorporate local traditions and beliefs into the management of mental disorders.

\subsection{Patients, Their Families, Their Employers, Health Care Workers, Educators and the Community}

In order to promote education and public awareness, the programmes on mental 
disorders should be directed at the IHPs, patients their families, their employers, health care workers, educators and the community. The community needs to be educated about mental disorders, and the signs and symptoms. This can help with early detection of mental disorders at community level and self-referral to IHPs or clinic. The involvement of family members, people with mental disorders and the community at large is important in order to regulate the management of mental health disorders at full scale. Everyone should know about the nature and symptoms of mental disorders. Workshops with all local stakeholders, i.e. faith healers, relatives and friends, herbalists, diviners, and nurses are encouraged in order to ensure the sustainability of mental health care.

The training material need to highlight the nature of mental disorders, signs and symptoms, treatment, etc from both approaches. The training material also need to include the attitude, knowledge and perceptions of the Zimbabwean people towards mental disorders. The material should cover the partnership between IHPs and BTPs, including the cultural environment. Information dissemination initiative for indigenous practices is also recommended. This will enable various stakeholders to have an appreciation on the cultural nuances of IH about mental disorders. The paper recommend that all these must be backed with a policy on the partnership between IHPs with BTPs in Zimbabwe.

\subsection{Policy}

The paper has revealed that the IHPs should be recognized and integrated with the BTPs in mental health treatment so that there is inclusion of indigenous healing characteristics in order to address patients' physical, mental and spiritual/cultural needs. The government of Zimbabwe should create a department under the mental health dedicated for IH systems of health. The ministry of health is recommended to partner with the ministry of education, non-governmental organisations to ensure effective dissemination of information regarding mental health conditions in terms of their nature and effects in life from both perspectives, IH and BT. The paper, therefore recommend the formation of partnership between IHPs and BTPs. The partnership will help to improve on holistic surveillance of mental disorders in Zimbabwe. This is because IHPs are readily available and cheap and naturally committed to their calling. The policy makers should develop the policies and publish these policies in the national guidelines to incorporate the cultural and the spiritual symptoms in the management of mental disorders.

\subsection{Research}

Future research must focus on integrating IHPs with BTPs in order to improve on the surveillance work and management of mental disorders.

\section{Conflicts of Interest}

The authors declare no conflicts of interest regarding the publication of this paper. 


\section{References}

[1] Charlson, F.J., Diminic, S. and Whiteford, H.A. (2015) The Rising Tide of Mental Disorders in the Pacific Region: Forecasts of Disease Burden and Service Requirements from 2010 to 2050: The Rising Tide of Mental Disorders in the Pacific Region. Asia \& the Pacific Policy Studies, 2, 280-292. https://doi.org/10.1002/app5.93

[2] Weine, S., Danieli, Y., Silove, D., Ommeren, M.V., Fairbank, J.A. and Saul, J. (2002) Guidelines for International Training in Mental Health and Psychosocial Interventions for Trauma Exposed Populations in Clinical and Community Settings. Psychiatry, 65, 156-164. https://doi.org/10.1521/psyc.65.2.156.19936

[3] Minas, H., Wright, A. and Kakuma, R. (2014) Goals and Organisational Structure of the Movement for Global Mental Health. International Journal of Mental Health Systems, 8, 31. https://doi.org/10.1186/1752-4458-8-31

[4] Upchurch, D.M. and Rainisch, B.K.W. (2013) A Sociobehavioral Model of Use of Complementary and Alternative Medicine Providers, Products, and Practices: Findings from the 2007 National Health Interview Survey. Journal of EvidenceBased Complementary \& Alternative Medicine, 18, 100-107. https://doi.org/10.1177/2156587212463071

[5] Campbell, B. (2000) Whose Knowledge? Indigenous Views on the Terms of Development Participation. John Wiley and Sons, New York.

[6] Oliver, S.J. (2013) The Role of Traditional Medicine Practice in Primary Health Care within Aboriginal Australia: A Review of the Literature. Journal of Ethnobiology and Ethnomedicine, 9, 46. https://doi.org/10.1186/1746-4269-9-46

[7] Shoko, T. (2008) Karanga Traditional Medicine and Healing. African Journal of Traditional, Complementary and Alternative Medicines, 4, 501-509. https://doi.org/10.4314/ajtcam.v4i4.31244

[8] Sandlana, N. and Mtetwa, D. (2008) African Traditional and Religious Faith Healing Practices and the Provision of Psychological Wellbeing among amaXhosa People. Indilinga: African Journal of Indigenous Knowledge Systems, 7, 119-131. https://doi.org/10.4314/indilinga.v7i2.26430

[9] Patel, V. (2007) Mental Health in Low- and Middle-Income Countries. British Medical Bulletin, 81, 81-96. https://doi.org/10.1093/bmb/ldm010

[10] Michaud, C.M., Murray, C.J. and Bloom, B.R. (2001) Burden of Disease-Implications for Future Research. JAMA, 285, 535-539.

https://doi.org/10.1001/jama.285.5.535

[11] Watts, S.C., Bhutani, G.E., Stout, I.H., Ducker, G.M., Cleator, P.J., McGarry, J. and Day, M. (2002) Mental Health in Older Adult Recipients of Primary Care Services: Is Depression the Key Issue? Identification, Treatment and the General Practitioner. International Journal of Geriatric Psychiatry, 17, 427-437.

https://doi.org/10.1002/gps.632

[12] Patel, V., Araya, R., Chatterjee, S., Chisholm, D., Cohen, A., De Silva, M., van Ommeren, M., et al. (2007) Treatment and Prevention of Mental Disorders in Low-Income and Middle-Income Countries. The Lancet, 370, 991-1005.

https://doi.org/10.1016/S0140-6736(07)61240-9

[13] Chitindingu, E., George, G. and Gow, J. (2014) A Review of the Integration of Traditional, Complementary and Alternative Medicine into the Curriculum of South African Medical Schools. BMC Medical Education, 14, 40. https://doi.org/10.1186/1472-6920-14-40

[14] Chiwanza, K., Musingafi, C.C. and Mupa, P. (2013) Challenges in Preserving In- 
digenous Knowledge Systems: Learning from Past Experiences. Information and Knowledge Management, 3, 19-25.

[15] Cohen, A., Organization, W.H., Organization, W.H., et al. (2001) The Effectiveness of Mental Health Services in Primary Care: The View from the Developing World. Mental Health Policy and Service Development, Department of Mental Health and Substance Dependence, Noncommunicable Diseases and Mental Health, World Health Organization.

[16] Pienaar, A. (2014) Mental Health Care in Africa: An Evidence-Based Approach. https://www.google.com/search?client=firefox

[17] Cross, T.L. (2003) Culture as a Resource for Mental Health. Cultural Diversity and Ethnic Minority Psychology, 9, 354. https://doi.org/10.1037/1099-9809.9.4.354

[18] Patel, V., Simunyu, E. and Gwanzura, F. (1997) The Pathways to Primary Mental Health Care in High-Density Suburbs in Harare, Zimbabwe. Social Psychiatry and Psychiatric Epidemiology, 32, 97-103. https://doi.org/10.1007/BF00788927

[19] Sodi, T. and Bojuwoye, N. (2011) Cultural Embeddedness of Health, Illness and Healing: Challenges for Integrating Traditional Healing and Western Oriented Health Care Systems. Journal of Psychology in Africa, 21, 349-356. https://doi.org/10.1080/14330237.2011.10820467

[20] Steel, Z., Marnane, C., Iranpour, C., Chey, T., Jackson, J.W., Patel, V. and Silove, D. (2014) The Global Prevalence of Common Mental Disorders: A Systematic Review and Meta-Analysis 1980-2013. International Journal of Epidemiology, 43, 476-493. https://doi.org/10.1093/ije/dyu038

[21] Gberie, L. (n.d.) Mental Illness: Invisible But Devastating. Africa Renewal Online. http://www.un.org/africarenewal/magazine/december-2016-march-2017/mental-ill ness-invisible-devastating

[22] Dana, R.H. (2012) Mental Health Services for African Americans: A Cultural/Racial Perspective. Journal of Human Growth and Development, 9, 63-73.

http://www.revistas.usp.br/jhgd/article/download/39479/42363

[23] Jenkins, R., Othieno, C., Ongeri, L., Sifuna, P., Ongecha, M., Kingora, J., Ogutu, B., et al. (2015) Common Mental Disorder in Nyanza Province, Kenya in 2013 and Its Associated Risk Factors-An Assessment of Change since 2004, Using a Repeat Household Survey in a Demographic Surveillance Site. BTC Psychiatry, 15, 309. https://doi.org/10.1186/s12888-015-0693-5

[24] Husain, N., Mukherjee, I., Notiar, A., Alavi, Z., Tomenson, B., Hawa, F., Chaudhry, N., et al. (2016) Prevalence of Common Mental Disorders and Its Association with Life Events and Social Support in Mothers Attending a Well-Child Clinic: Findings from Mombasa, Kenya. SAGE Open, 6, Article ID: 215824401667732. https://doi.org/10.1177/2158244016677324

[25] Ndetei, D.M., Khasakhala, L.I., Kuria, M.W., Mutiso, V.N., Ongecha-Owuor, F.A. and Kokonya, D.A. (2009) The Prevalence of Mental Disorders in Adults in Different Level General Medical Facilities in Kenya: A Cross-Sectional Study. Annals of General Psychiatry, 8, 1. https://doi.org/10.1186/1744-859X-8-1

[26] Faydi, E., Funk, M., Kleintjes, S., Ofori-Atta, A., Ssbunnya, J., Mwanza, J., Flisher, A., et al. (2011) An Assessment of Mental Health Policy in Ghana, South Africa, Uganda and Zambia. Health Research Policy and Systems, 9, 17. https://doi.org/10.1186/1478-4505-9-17

[27] Ojukwu, M., Mbizo, J., Leyva, B., Olaku, O. and Zia, F. (2015) Complementary and Alternative Medicine Use among Overweight and Obese Cancer Survivors in the United States. Integrative Cancer Therapies, 14, 503-514. 
https://doi.org/10.1177/1534735415589347

[28] Ovuga, E., Boardman, J. and Oluka, E.G. (1999) Traditional Healers and Mental Illness in Uganda. Psychiatric Bulletin, 23, 276-279. https://doi.org/10.1192/pb.23.5.276

[29] Iheanacho, T., Kapadia, D., Ezeanolue, C.O., Osuji, A.A., Ogidi, A.G., Ike, A., Ezeanolue, E.E., et al. (2016) Attitudes and Beliefs about Mental Illness among Church-Based Lay Health Workers: Experience from a Prevention of Mother-toChild HIV Transmission Trial in Nigeria. International Journal of Culture and Mental Health, 9, 1-13. https://doi.org/10.1080/17542863.2015.1074260

[30] Baxter, A.J., Patton, G., Scott, K.M., Degenhardt, L. and Whiteford, H.A. (2013) Global Epidemiology of Mental Disorders: What Are We Missing? PLoS ONE, 8, e65514. https://doi.org/10.1371/journal.pone.0065514

[31] Flisher, A.J., Lund, C., Funk, M., Banda, M., Bhana, A., Doku, V., Green, A., et al. (2007) Mental Health Policy Development and Implementation in Four African Countries. Journal of Health Psychology, 12, 505-516. https://doi.org/10.1177/1359105307076237

[32] Chibanda, D., Cowan, F., Gibson, L., Weiss, H.A. and Lund, C. (2016) Prevalence and Correlates of Probable Common Mental Disorders in a Population with High Prevalence of HIV in Zimbabwe. BTC Psychiatry, 16, 55. https://doi.org/10.1186/s12888-016-0764-2

[33] Kidia, K., Machando, D., Mangezi, W., Hendler, R., Crooks, M., Abas, M., Jack, H., et al. (2017) Mental Health in Zimbabwe: A Health Systems Analysis. The Lancet Psychiatry, 4, 876-886. https://doi.org/10.1016/S2215-0366(17)30128-1

[34] Hendler, R., Kidia, K., Machando, D., Crooks, M., Mangezi, W., Abas, M., Jack, H., et al. (2016) "We Are Not Really Marketing Mental Health": Mental Health Advocacy in Zimbabwe. PLoS ONE, 11, e0161860. https://doi.org/10.1371/journal.pone.0161860

[35] Chibanda, D. (2017) How a Community-Based Approach to Mental Health Is Making Strides in Zimbabwe.

http://theconversation.com/how-a-community-based-approach-to-mental-health-is -making-strides-in-zimbabwe-79312

[36] Tibaijuka, A.K. (2005) Report of the Fact-Finding Mission to Zimbabwe to Assess the Scope and Impact of Operation Murambatsvina. UN, Harare.

[37] Chibanda, D., Mesu, P., Kajawu, L., Cowan, F., Araya, R. and Abas, M.A. (2011) Problem-Solving Therapy for Depression and Common Mental Disorders in Zimbabwe: Piloting a Task-Shifting Primary Mental Health Care Intervention in a Population with a High Prevalence of People Living with HIV. BTC Public Health, 11, 828. https://doi.org/10.1186/1471-2458-11-828

[38] Reeler, A.P., Mbape, P., Matshona, J., Mhetura, J. and Hlatywayo, E. (2001) The Prevalence and Nature of Disorders Due to Torture in Mashonaland Central Province, Zimbabwe. Torture, 11, 4-9.

[39] Peltzer, K., Pengpid, S., Puckpinyo, A., Yi, S. and Vu Anh, L. (2016) The Utilization of Traditional, Complementary and Alternative Medicine for Non-Communicable Diseases and Mental Disorders in Health Care Patients in Cambodia, Thailand and Vietnam. BTC Complementary and Alternative Medicine, 16, 92. https://doi.org/10.1186/s12906-016-1078-0

[40] Broadhead, J.C. and Abas, M.A. (1998) Life Events, Difficulties and Depression among Women in an Urban Setting in Zimbabwe. Psychological Medicine, 28, 29-38. https://doi.org/10.1017/S0033291797005618 
[41] Janz, N.K. and Becker, M.H. (1984) The Health Belief Model a Decade Later. Health Education Quarterly, 11, 1-47. https://doi.org/10.1177/109019818401100101

[42] Glaser, B.G. and Strauss, A.L. (2009) The Discovery of Grounded Theory: Strategies for Qualitative Research. Transaction Publishers, Piscataway.

https://books.google.com/books?hl=en\&lr=\&id=rtiNK68Xt08C\&oi=fnd\&pg=PP1\& $\mathrm{dq}=$ The+discovery+of+grounded+theory: + Strategies + for + qualitative+research\&ots $=$ UVwUTlVJZP\&sig=Su95_tyfwesd8nXv9dja3ZuX7Ao

[43] Ritchie, J., Lewis, J., Lewis, P., Nicholls, C.M. and Ormston, R. (2013) Qualitative Research Practice: A Guide for Social Science Students and Researchers. SAGE, Thousand Oaks.

[44] Pope, C. and Mays, N. (2013) Qualitative Research in Health Care. John Wiley \& Sons, Hoboken.

[45] Kumar, S. and Phrommathed, P. (2005) Research Methodology. In: Kumar, S. and Phrommathed, P., Eds., New Product Development, Springer, Berlin, 43-50. http://link.springer.com/content/pdf/10.1007/0-387-23273-7_3.pdf

[46] Parahoo, K. (2014) Nursing Research: Principles, Process and Issues. Palgrave Macmillan, London.

[47] Paltved, C. and Musaeus, P. (2012) Qualitative Research on Emergency Medicine Physicians: A Literature Review. International Journal of Clinical Medicine, 3, 772 789. https://doi.org/10.4236/ijcm.2012.37A136

[48] Huberman, A. and Miles, M. (2002) The Qualitative Researcher's Companion. SAGE Publications, Thousand Oaks. https://doi.org/10.4135/9781412986274

[49] LeCompte, M.D. and Schensul, J.J. (2010) Designing and Conducting Ethnographic Research. Rowman \& Littlefield, Lanham.

https://books.google.com/books?hl=en\&lr=\&id=xa7oIOlCUGwC\&oi=fnd\&pg=PR5 $\& \mathrm{dq}=$ Designing+and+conducting + ethnographic+research\&ots=n_ryshLuck\&sig $=\mathrm{q}$ o9ymoTOj_PzbCW9-vOmhD_i0EM

[50] Patton, M.Q. (2000) Qualitative Research \& Evaluation Methods. 3rd Edition, Sage Publications, London.

[51] Robertson, B.A. (2006) Does the Evidence Support Collaboration between Psychiatry and Traditional Healers? Findings from Three South African Studies. African Journal of Psychiatry, 9, 87-90. https://doi.org/10.4314/ajpsy.v9i2.30210

[52] Agara, A.J., Makanjuola, A.B. and Morakinyo, O.B. (2008) Management of Perceived Mental Health Problems by Spiritual. African Journal of Psychiatry, 11, 113-118. https://doi.org/10.4314/ajpsy.v11i2.30262

[53] Atilola, O. (2016) Mental Health Service Utilization in Sub-Saharan Africa: Is Public Mental Health Literacy the Problem? Setting the Perspectives Right. Global Health Promotion, 23, 30-37. https://doi.org/10.1177/1757975914567179

[54] Lopez, R.A. (2005) Use of Alternative Folk Medicine by Mexican American Women. Journal of Immigrant Health, 7, 23-31. https://doi.org/10.1007/s10903-005-1387-8

[55] Helwig, D. (2010) Traditional African Medicine. Encyclopedia of Alternative Medicine.

[56] Chavunduka, G.L. (1994) Traditional Medicine in Modern Zimbabwe. University of Zimbabwe Publications, Zimbabwe, 115 p.

https://www.worldcat.org/title/traditional-medicine-in-modern-zimbabwe/oclc/323 $\underline{12472}$

[57] Gunda, M.R. (2007) Christianity, Traditional Religion, and Healing in Zimbabwe: 
Exploring the Dimensions and Dynamics of Healing among the Shona. Swedish Missiological Themes, 95, 232.

[58] Patel, V., Abas, M., Broadhead, J., Todd, C. and Reeler, A. (2001) Depression in Developing Countries: Lessons from Zimbabwe. BT): British Medical Journal, 322, 482. https://doi.org/10.1136/bmj.322.7284.482

[59] Berg, A. (2004) Ubuntu-A Contribution to the Civilization of the Universal. In: Singer, T. and Kimbles, S.L., Eds., The Cultural Complex. Contemporary Jungian Perspectives on Psyche and Society, Brunner-Routledge, New York, 239-250.

[60] Hastwell (2014) Aboriginal Healers Working to Keep Traditional Medicine Alive. Radio National.

https://www.abc.net.au/radionational/programs/awaye/aboriginal-healers-workingto-keep-traditional-medicine-alive/5471058

[61] Lo, H.-T. and Fung, K.P. (2003) Culturally Competent Psychotherapy. Canadian Journal of Psychiatry, 48, 161-170. https://doi.org/10.1177/070674370304800304

[62] Ngoma, M.C., Prince, M. and Mann, A. (2003) Common Mental Disorders among Those Attending Primary Health Clinics and Traditional Healers in Urban Tanzania. The British Journal of Psychiatry: The Journal of Mental Science, 183, 349-355. https://doi.org/10.1192/bjp.183.4.349 